\title{
Targeting BMP signaling in the bone marrow microenvironment of Myeloid Leukemia
}

Sylvain Lefort ${ }^{1,2,3,4}$ and Véronique Maguer-Satta ${ }^{1,2,3,4}$

1-CNRS UMR5286, Centre de Recherche en Cancérologie de Lyon, 69000 Lyon;

2-Inserm U1052, Centre de Recherche en Cancérologie de Lyon, 69000 Lyon;

3-Université de Lyon, 69000, Lyon, France;

4-Department of Signaling of tumor escape, Lyon

Corresponding author: Dr. Véronique MAGUER-SATTA, PhD, Cancer Research Center of LyonCRCL, U1052-UMR5286, 28 rue Laennec, 69373 Lyon Cedex 08, FRANCE, 33-478 782 907, Fax 33478782 720, veronique.maguer-satta@lyon.unicancer.fr

Keywords: BMP2/4, BMPR1, niche, resistance, persistence, leukemic stem cells, targeted therapies, CML, AML.

Counts: text: 2854 words; 1 figure, and 51 references 


\section{Abstract}

The BMP pathway regulates the fate and proliferation of normal hematopoietic stem cells (HSC) as well as interactions with their niche. While BMP2 and BMP4 promote HSC differentiation, only BMP4 maintains HSC pool and favors interactions with their niche. In myeloid leukemia, we have identified intrinsic and extrinsic dysregulations of the BMP pathway in Chronic Myeloid Leukemia (CML) and Acute Myeloid leukemia (AML) responsible for leukemic stem cells (LSC) survival. In AML, BMP pathway alterations sustain and promote resistant immature-like leukemic cells by activating a new signaling cascade. Binding of BMP4 to BMPR1A leads to $\triangle N p 73$ expression, which in turn induces NANOG, altogether associated with a poor patient's prognosis. Despite efficient targeted therapies, like Tyrosine Kinase Inhibitors (TKI) in CML, many patients retain LSCs. Our laboratory demonstrated that the BMP pathway sustains a permanent pool of LSCs expressing high levels of BMPR1B receptor, that evolve upon treatment to progressively implement a BMP4 autocrine loop, leading to TKIresistant cells. Single cell RNA-Seq analysis of TKI-persisting LSCs showed a co-enrichment of BMP with Jak2-signaling, quiescence and stem cell (SC) signatures. Using a new model of persisting LSCs, we recently demonstrated that BMPR1B+ cells display co-activated Smad1/5/8 and Stat3 pathways and could be targeted by blocking BMPR1B/Jak2 signal. Lastly, a specific BMPR1B inhibitor impaired BMP4-mediated LSC protection against TKIs. Altogether, data based on various studies including ours, indicate that BMP targeting could eliminate leukemic cells within protective bone marrow microenvironment to efficiently impact residual resistant or persistent of LSCs in myeloid leukemia. 
Cancer is a heterogeneous disease with co-existence of different populations of cancer cells within the same tumor. Some of them have properties that closely resemble normal stem cells (SC), which gave rise to the concept of cancer stem cells (CSCs) (1). Aside from the definition of the origin of the CSC population, the reciprocal influences of tumor cells and their environment appears to be a key element to understand their biology, in order to design more efficient treatments to avoid recurrence $(2,3)$. Targeting the dialogue between cancer cells and their niche, by taking advantage of differences in the biology of normal and CSCs, such as differences in surface phenotype, self-renewal/quiescence and stem cell-niche interactions, should allow successful CSC targeting and to improve patient outcome (3). Many signaling pathways have consistently been explored, although no major breakthrough has been made. Among the main pathways that participate in the permanent and dynamic dialogue between the bone marrow microenvironment and resident stem cells, the Bone Morphogenetic Proteins (BMP) signaling constitutes a major bi-directional actor. Indeed, soluble BMP molecules, members of the TGF $\beta$ superfamily, govern SC regulation during development or in adult tissues including hematopoietic (4), neural and epithelial systems (5) both directly and indirectly by affecting their niche (6-11). Alterations of this pathway have been shown to play a role in cell transformation and progression, in some cases by promoting CSC properties in solid tumors (11-13) as well as in leukemia (14). However, most studies have mainly documented events related to advanced stages. BMP ligands control differentiation, angiogenesis, immune response and stromal cell regulation indicating that they could participate in tumor initiation and promote CSC expansion and survival (8). One of the main alterations identified affects BMP signaling type 1 serine-threonine receptors responsible for BMP signal transduction through a cascade of protein phosphorylation. Indeed, BMP2 and BMP4 have emerged as key regulators of normal SCs and CSCs, in particular in the hematopoietic system. The importance of the BMP signaling pathway at very early steps of transformation has been evaluated in representative models of CSC in the hematopoietic system including Chronic Myeloid Leukemia-CML (the reference model for SC transformation) 
and Acute Myeloid Leukemia-AML and breast cancer (representing a solid tumor model containing CSCs, but of debatable origin). In these systems, using primary cells and cellular models of CSCs mimicking early stages of transformation, we revealed major dysregulations of the BMP pathway in SCs and their niche at diagnosis, and showed their direct implication in the emergence and expansion of CSCs. To further test the importance of this pathway to target cancer (leukemic) stem cells (LSCs), the spatio-temporal setting of mechanisms underlying resistance remains to be defined and constitutes a major therapeutic challenge to achieve complete recovery through eradication of persistent LSCs. Here, this review highlights the dynamic alterations of the BMP signaling that occurs during disease progression and which currently remained the most investigated in CML model.

\section{Initiation of BMP signaling alterations in stem cells and their niche}

We have demonstrated in CML that transformation of normal hematopoietic SCs (HSCs) by the chromosomal translocation $t(9,22)$ leading to the expression of the fusion protein $B C R$ $A B L$, results in a profound alteration of BMP signaling. These alterations highlight a true dysregulation of this signaling pathway as illustrated by the higher expression of BMPRIB in almost all CML subpopulations at diagnosis at both transcriptional and protein levels. BMPRIB over-expression constitutes an early event in disease progression and is a direct consequence of the introduction of BCR-ABL in immature CD34+ cells (TF1 model) (15). Alterations of the BMP pathway in CML CD34+ cells do not prevent leukemic cells from responding to extracellular stimulation by BMP2/4 but are correlated with the maintenance and amplification of LSCs in response to BMP4. Importantly, BMP4-expanded SCs were all genotypically identified as leukemic cells (Philadelphia Chromosome+, $\mathrm{Ph}+$ ), suggesting that the biological response of CD34+ cells to BMP2/4 is specifically altered in CML. This contributes to an increased sensitivity of $\mathrm{Ph}+$ cells to external BMP signals (15).

While intrinsic genetic alterations are clearly very important, data obtained over the past 15 years argue in favor of a complementary and synergistic role for extrinsic signals that actively participate in the transformation process as well as to promote cancer progression and 
treatment escape mechanisms $(3,14,16)$. Remarkably, the donor-induced leukemia constitutes an ultimate proof of that concept. Indeed, in some cases, the allogenic donor remains healthy while the leukemic patient transplanted with cells from this donor develop a new leukemia that results from transformation of these donor-engrafted cells. Moreover, this de-novo leukemia can be similar or different from the initial one that constituted the clinical indication for donor cell transplantation (17). Therefore, as all hematopoietic cells have been eradicated by radiation during patient conditioning prior to the graft, the only remaining altered signal is likely to come from the patient bone marrow microenvironment itself. More recently, single cell transcriptomic data revealed that BCR-ABL -negative HSC in CML patients are transcriptionally altered when compared to normal donor HSC at the single cell level (18). This strengthen how the BM niche changes which occur in leukaemia can influence normal stem cell behavior.

The dynamic and reciprocal dialogue between SCs and their niche is thus of utmost importance for their maintenance and regulation.

In this context, a strong increase in soluble BMP2 and BMP4 levels was measured in the tumoral niche of CML patients (15) and was shown to be produced by the SC niche, often dysregulated without being transformed per se (3). Indeed, the expression of BMP ligand by leukemic cells was lower (15), and, BMP2 for example has been identified as the most repressed gene at the transcriptional level in CML LSCs (19). Surprisingly, similar downregulation of BMP2 has been reported at diagnosis in luminal breast cancer (11), suggesting a common event related to early steps of cell transformation.

At the functional level, these intrinsic and extrinsic alterations of the BMP pathway constitute major events in transformation of immature cells that contribute to sustaining a permanent pool of LSCs and progenitors expressing high levels of the BMPR1B receptor in CML $(4,15,20)$. Consistently, a prolonged expression of BCR-ABL in SCs (CML model from TF1 cells) leads to profound changes in DNA replication by changing replication fork features during tumor progression (21). These sets of data arising from different studies indicate that, in chronic phase CML, LSCs display a hyper-sensitivity of CD34+ CML cells to soluble BMP ligands 
abnormally abundant in the bone marrow niche leading to the over amplification of the myeloid compartment, a hallmark of this disease.

\section{BMP signaling is a key pathway to reprogram myeloid leukemic cells in stem-like cells} AML is the first model in which the existence of LSCs was established, leading to the identification of CSCs in numerous hematological malignancies and solid tumors (1). AML is a very complex pathology that displays a large number of distinct genetic alterations linked to the development of this disease, arising in some cases from CML progression. Similarly to what we observed in CML, we have revealed functional alterations of the BMP pathway in AML at diagnosis (22). Conversely to CML for which BMPR1B (Alk6) was identified as the most altered receptor, BMPR1A (Alk3) appears to be the main dysregulated receptor in bone marrow or peripheral blood blast cells isolated from adult AML (22). Furthermore, similarly to CML and breast cancer, higher levels of soluble BMP ligands were also detected, mainly BMP4 for AML (22). Despite the fact that various AML hematopoietic cell lines have been reported to produce BMP4 and BMP2 transcripts (23), primary AML mononuclear cells showed a decrease in BMP2 and BMP4 transcript (22). However, further investigations are required to determine the origin of high levels of soluble BMP4 protein measured in the bone marrow leukemic niche (22). However, in AMKL, an aggressive form of pediatric AML, appearance of a specific fusion protein CBFA2T3-GLIS2 leads to a drastic overexpression of BMP2 and BMP4, by leukemic cells associated with colony formation capacities, the self-renewal and functional capacities of LSCs (24). This confirms that alterations of the BMP signaling is recurrent in early steps of cancer and also indicates that some specificity related to developmental stage can occur. Further investigations will be required to decipher functional implications of these specificities between adult and pediatric leukemia.

At the functional level, a new signaling cascade linking BMP4 binding to BMPR1A was revealed to imprint immature-like properties to $A M L$ blasts through $\triangle N p 73$ and NANOG regulation (22). Very interestingly, it was previously reported that murine fibroblasts could be 
efficiently reprogrammed into myeloid and lymphoid cells through extracellular BMP signaling involving BMP4 (25). Therefore, such imbalance in BMP4 level may lead to favor an immaturelike phenotype of more mature AML transformed cells (22). These findings imply that alteration of BMP signaling can occur not only in the immature cell compartment but also in more differentiated myeloid cells, enhancing stem-cell features of leukemic cells.

\section{Dynamics of BMP signaling alterations under the pressure of treatment}

Numerous studies have focused on the identification of intrinsic hematopoietic cell resistance (or persistence) mechanisms to explain rare primary resistance, though relapse occurs more frequently after an initial hematologic, cytogenetic and even molecular response. In $30-40 \%$ of cases, no obvious resistance mechanism to TKI could be identified in CML, including pharmacological ones. During the last decade, consistent demonstrations revealed the critical role of the HSC niche in the development of hematopoietic tumors and their maintenance throughout time $(15,26-32)$. Although the microenvironment is dissimilar in different tumors, involving multiple cell types and proteins, it may exert and pervert tumor cells and favor their resistance to treatments. This phenomenon is emerging as a new paradigm to explain resistance of solid tumors (33).

A growing body of evidence suggests that molecular and functional alterations evolve upon treatment. Based on recent technological breakthroughs, several SCs subpopulations have been identified in CML, with distinct and dynamic proportions at diagnosis and following TKI treatment $(18,34)$. This raised the hypothesis that resistance could emerge from the heterogeneity of LSC subpopulations depending on the cues delivered by their microenvironment. As several studies have demonstrated that BMP pathway alterations play a fundamental and recurrent role in early stages of SC transformation $(4,14,15,20,22,24)$, this suggests that this signaling is most likely to be involved in resistance of leukemic cells and especially of LSCs. This concept was formally established by analyzing bone marrow samples of LSCs and progenitors isolated from patients either sensitive or resistant to TKI, which showed that several elements of the BMP and TGF signaling pathways remained highly 
modified or evolved upon treatment and were correlated with LSC survival (35-37). In CML resistant cells, a specific molecular signature based on dysregulated genes from the TGF and BMP signaling in mature and immature cells was correlated with a poor response to TKI treatment (36). Earlier studies have identified in both primary cells and CML cell lines the abnormal expression of the embryonic transcription factor Twist-1 as an actor of CML TKIresistance, especially in LSCs $(38,39)$. It is now established that LSCs adapt to targeted therapy by implementing a BMP4 autocrine loop simultaneously to a sustained and increased dysregulation of the LSC niche upon long-term treatment (37). This leads to a further increase in BMPR1B expression that upon BMP4 signal induces the upregulation of Twist-1 expression, altogether protecting LSCs from TKI-driven cell death (37).

Most AML patients achieve complete remission after intensive induction chemotherapy, but the majority of them relapse within several years. Recurrence may reflect the survival of resistant immature-like LSCs after chemotherapy (40). In AML leukemic cells, a signaling cascade involving BMP4 binding to BMPR1A drive $\triangle N p 73$ and NANOG expression to sustained an immature-like phenotype. It also identified potential markers to predict relapse of AML patients at diagnosis and explained survival and features of immature bone marrow AML cells (22). A previous study highlighted the importance of the BMP pathway in adult AML, using various cell lines (41). Interestingly, BMP4 controls the expression of the MIXL1 gene, aberrantly expressed in AML and involved in early steps of leukemogenesis in a mouse model (42). The predictive value of BMPR1 and downstream targets suggests that targeting the BMP pathway could be of interest in adult AML. Indeed, BMP type-1 receptors inhibition has been shown to efficiently impact MIXL1-expressing AML cells (41). Collectively, these data support that targeting the BMP pathway could constitute a new therapeutic tool to decrease the progenitor capacity of LSCs $(24,43)$.

\section{Multi-targeting of BMP signaling impairs persistent LSC survival in their leukemic ecosystem}

Eradication of LSCs then raises many hopes in the treatment of cancer but remains a challenge 
justifying the importance for better understanding the mechanisms underlying LSC persistence and survival under treatment. Recent CML single-cell level analysis revealed the selective persistence of a distinct and highly quiescent CML LSC subset already present at diagnosis (18). Indeed, in chronic phase, regardless of patient response to TKI, a fraction of CML LSCs survive, sustained by interactions with their niche responsible for disease persistence and relapse following treatment cessation due to the implementation of resistance mechanisms $(44,45)$. We have recently investigated downstream signaling involved in LSC persistence in patients under TKI treatment and identified a sub-fraction of LSCs that specifically co-activate the BMP/BMPR1B and Jak2/Stat3 signaling in bone marrow of CML patients in remission following treatment. Using a model of persisting LSCs, the importance of the BMP signaling in SC dormancy driven by its tumor microenvironment was identified as responsible for disease persistence in response as an adaptation to treatment (46). When CML CD34 ${ }^{+}$-cells persist under continuous TKI-treatment, all BMPR1B+ cells co-activate Smad1/5/8 and Stat3 pathways. These cells could then be efficiently targeted by blocking BMPR1B/Jak2 signaling using the BMPR-1B inhibitor (E6201) that triggers LSC death, and/or the JAK2/STAT3 inhibitor (AG490) (46). The importance of the JAK2 and Stat3 signaling in CML LSC persistence was previously reported by several groups (47-50). This underlines the importance of synergistic effects between BMP and Jak2 targeting in order to eradicate LSCs that persist in the tumor microenvironment, even in patients with apparent good treatment response (46). In the context of resistant CML cells, dual targeting using TKI and BMP inhibition efficiently reduce the number of LSCs and derived progenitors (36). This indicates that BMP-specific inhibitors could be efficient at different stages of disease progression including advanced CML that could transform into AML as previously discussed (41).

However, we have unveiled that only BMPR1B-expressing cells that closely interact with stromal cells of the leukemic niche enter quiescence in the presence of TKI treatment. Impairing the BMP4 survival signal provided by this niche releases LSCs from quiescence and allows them to enter differentiation. Importantly, the BMPR inhibitor alone is capable of simultaneously blocking BMP production by stromal cells surrounding LSCs while other current 
treatments by themselves are inefficient in doing so. In addition, in AML, soluble BMPs induce Smad1/5 signaling in the bone marrow stromal cells to control their osteogenic differentiation suggesting that targeting of the BMP signaling could not only target leukemic cells (41) but also could contribute to restore normal MSC differentiation (23). Indeed, the monitoring of patients under TKI treatment for almost a decade has clearly highlighted that alteration of the leukemic niche in CML is not corrected by current treatments in either resistant (37) or sensitive CML patients (46).

\section{Conclusion}

The current paradigm of leukemogenesis implies a multistep process that involves different types of genetic alterations with no obvious hierarchy and understanding of the sequential clonal selection (51). In this context, crosstalk between LSCs and the associated bone marrow stroma appears as an essential relationship for leukemic progression, and response to therapy $(52,53)$. Moreover, crosstalk between BMP signaling (either canonical/Smad dependent or non-canonical/Smad independent) with other pathways emerge as an important parameter to decipher how BMP-signaling constitute a complex and dynamic molecular platform at the interplay between hematopoietic cells and their surrounding microenvironment during leukemia progression (54). Following long-term continuous targeted therapy, LSCs adapt and escape through diverse mechanisms. BMP signaling is then likely to constitute a recurrent way for LSCs to persist and escape drug pressure while allowing them to foster slow disease progression. Both the progressive implementation of an intrinsic BMP autocrine loop and the permanent exogenous and continuous increase in dysregulations of BMP ligand production by the LSC microenvironment thus favor the continuous evolution and progress of CML into an accelerated phase in some cases leading to AML transformation. Therefore, despite relative efficient therapies, many patients suffering from myeloid leukemia retain persistent LSCs and would then benefit from their eradication within the remission period achieved by current therapies. To that aim, the BMP pathway appears to be an emerging important target constituting a regulator to sustain a permanent pool of LSCs expressing high levels of the type 
1 BMP receptor, that evolve upon treatment to further dysregulate resistant cells. Therefore, targeting both BMPR1 and the protective bone marrow microenvironment that provides BMP ligands could efficiently impact residual resistant or persistent LSCs at different stages of myeloid progression (Figure 1).

Altogether, several studies have demonstrated in various cancers that CSCs display a hypersensitivity to BMP signals that contribute to setting the tumor ecosystem and promoting dynamic remodeling. This pathway represents a very early and efficient target for the development of new therapeutic strategies. Importantly, in both myeloid leukemia $(15,22)$ and breast cancers (11), we highlighted the important functions of the BMP receptor type 1 , since its activation results in the amplification of a normal response to BMP in SCs and contributes to the survival of CSCs independently of the initial transforming event. Due to the broad involvement of BMP signaling in cancer, identification of recurrent alterations of this pathway is becoming an important common feature in different cancers. Indeed, it is increasingly evident that, in addition to their known role at late stages of the disease, BMP pathway alterations play a fundamental and recurrent role at early stages of SC, in their expansion and maintenance, treatment escape and tumor progression. Thus increasing evidence identifies this signaling pathway as a robust target to either eradicate LSCs early on or prevent their further progression.

\section{Perspectives}

(i) The adult bone marrow provides essential niches for hematopoietic stem cell (HSC) maintenance and differentiation to meet the demand of blood and immune cell production during homeostasis and stress.

(ii) The BMP pathway regulates the fate and proliferation of normal stromal and HSC and modulates their permanent dialogue. It is also an essential actor of leukemic stem cells (LSC) 
persistence and niche remodeling in Chronic Myeloid Leukemia (CML) and Acute Myeloid leukemia (AML).

(iii) As the BMP pathway is involved in regulating LSC and their niche from early stages of the disease to tumor escape and resistance to treatments, patients would benefit from specific targeting of the BMP signaling to achieve persistent LSC eradication and restore a normal bone marrow microenvironment.

\section{Funding and Acknowledgements}

We thank P. Battiston (Cytometry Facility, CRCL, Lyon) for her technical assistance, B. Manship for English editing. We thank Dr T. Myers from Spirita Oncology for providing us with E6201. Fundings from: patients' association LMC France and her president Mrs Mina Daban; "Fondation de France" 2014-0047501 and 2017-00076282/Fondation Ramona Ehrman Amador, "Association Laurette Fugain" ALF2014-03, Ligue contre le Cancer (Haute Savoie, Loire, Puy de Dôme and Rhone), "Association ALTE-SMP", the Institut Convergence PLASCAN grants to V.M-S; S.L. and/or F-E.N. PhD fellowship was obtained from "Ligue contre le cancer" (K.A). 


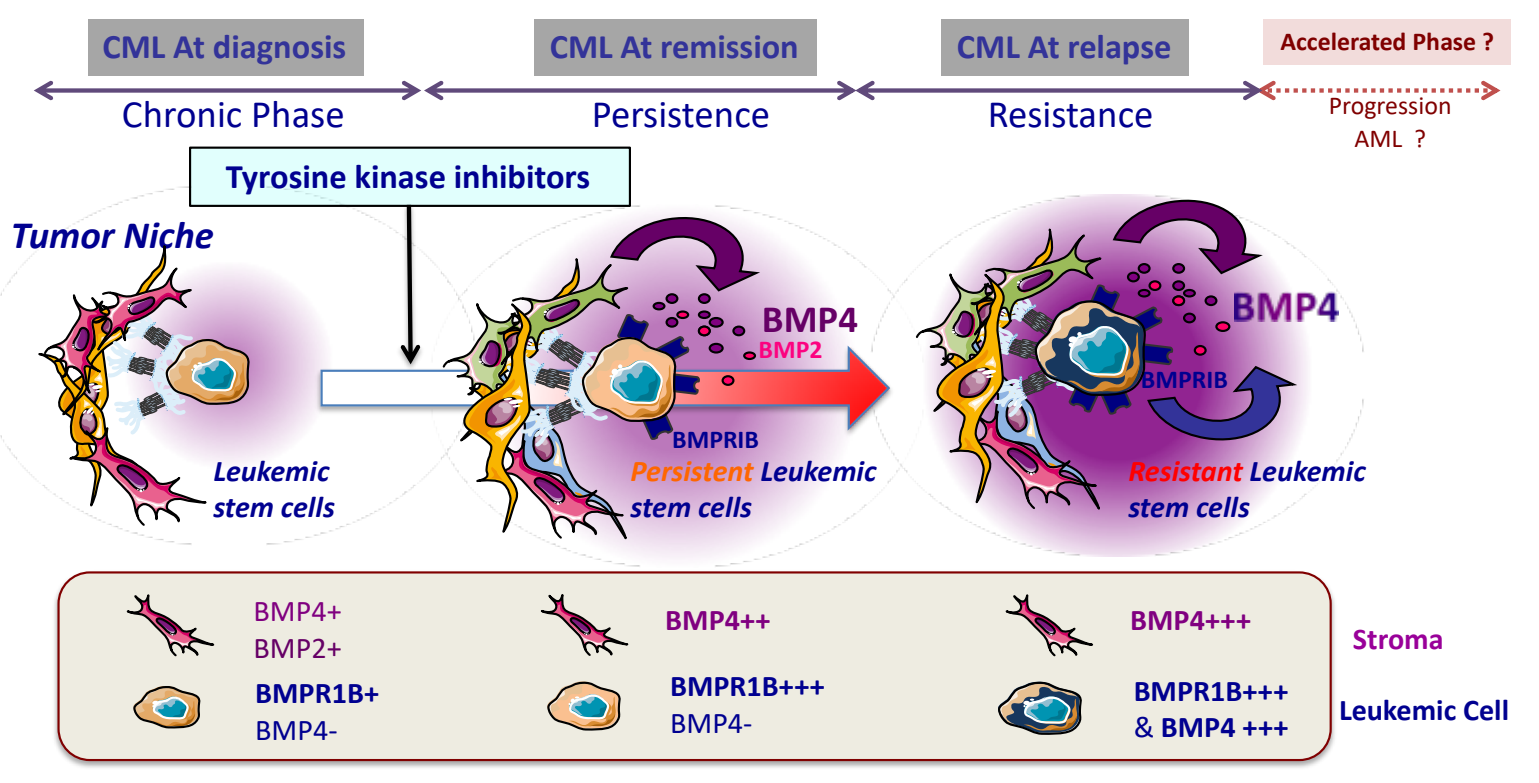

Figure 1: Evolution of BMP signaling alterations in the tumor ecosystem of CML, from transformation to treatment escape and progression toward AML. 
1. Bonnet D, Dick JE. Human acute myeloid leukemia is organized as a hierarchy that originates from a primitive hematopoietic cell. Nature Medicine. 1997;3(7):730-7.

2. Besancon R, Valsesia-Wittmann S, Puisieux A, Caron de Fromentel C, Maguer-Satta V. Cancer stem cells: the emerging challenge of drug targeting. Curr Med Chem. 2009;16(4):394-416.

3. Maguer-satta V. The stem cell niche: the black master of cancer. In: Stanley S, editor. Cancer stem cells. Intech: Intech; 2011. p. 215-40.

4. Toofan P, Wheadon $\mathrm{H}$. Role of the bone morphogenic protein pathway in developmental haemopoiesis and leukaemogenesis. Biochem Soc Trans. 2016;44(5):1455-63.

5. Rendl M, Polak L, Fuchs E. BMP signaling in dermal papilla cells is required for their hair follicle-inductive properties. Genes Dev. 2008;22(4):543-57.

6. Jeanpierre S, Nicolini FE, Kaniewski B, Dumontet C, Rimokh R, Puisieux A, et al. BMP4 regulation of human megakaryocytic differentiation is involved in thrombopoietin signaling. Blood. 2008;112(8):3154-63.

7. Zhang J, Niu C, Ye L, Huang $\mathrm{H}$, He X, Tong W-G, et al. Identification of the haematopoietic stem cell niche and control of the niche size. Nature. 2003;425(6960):836-41.

8. Pickup MW, Owens P, Moses HL. TGF-beta, Bone Morphogenetic Protein, and Activin Signaling and the Tumor Microenvironment. Cold Spring Harb Perspect Biol. 2017;9(5).

9. Katagiri T, Watabe T. Bone Morphogenetic Proteins. Cold Spring Harb Perspect Biol. 2016;8(6).

10. Clement F, Xu X, Donini CF, Clement A, Omarjee S, Delay E, et al. Long-term exposure to bisphenol A or benzo(a)pyrene alters the fate of human mammary epithelial stem cells in response to BMP2 and BMP4, by pre-activating BMP signaling. Cell Death Differ. 2017;24(1):155-66.

11. Chapellier M, Bachelard-Cascales E, Schmidt X, Clement F, Treilleux I, Delay E, et al. Disequilibrium of BMP2 levels in the breast stem cell niche launches epithelial transformation by overamplifying BMPR1B cell response. Stem Cell Reports. 2015;4(2):239-54.

12. Lee J, Son MJ, Woolard K, Donin NM, Li A, Cheng CH, et al. Epigenetic-mediated dysfunction of the bone morphogenetic protein pathway inhibits differentiation of glioblastoma-initiating cells. Cancer Cell. 2008;13(1):69-80.

13. Choi YJ, Ingram PN, Yang K, Coffman L, Iyengar M, Bai S, et al. Identifying an ovarian cancer cell hierarchy regulated by bone morphogenetic protein 2. Proc Natl Acad Sci U S A. 2015;112(50):E6882-8.

14. Zylbersztejn F, Flores-Violante M, Voeltzel T, Nicolini FE, Lefort S, Maguer-Satta V. The BMP pathway: A unique tool to decode the origin and progression of leukemia. Exp Hematol. 2018;61:36-44.

15. Laperrousaz B, Jeanpierre S, Sagorny K, Voeltzel T, Ramas S, Kaniewski B, et al. Primitive CML cell expansion relies on abnormal levels of BMPs provided by the niche and on BMPRIb overexpression. Blood. 2013;122(23):3767-77.

16. Gooding S, Olechnowicz SWZ, Morris EV, Armitage AE, Arezes J, Frost J, et al. Transcriptomic profiling of the myeloma bone-lining niche reveals BMP signalling inhibition to improve bone disease. Nat Commun. 2019;10(1):4533. 
17. Flynn CM, Kaufman DS. Donor cell leukemia: insight into cancer stem cells and the stem cell niche. Blood. 2007;109(7):2688-92.

18. Giustacchini A, Thongjuea S, Barkas N, Woll PS, Povinelli BJ, Booth CAG, et al. Single-cell transcriptomics uncovers distinct molecular signatures of stem cells in chronic myeloid leukemia. Nat Med. 2017;23(6):692-702.

19. Gerber JM, Gucwa JL, Esopi D, Gurel M, Haffner MC, Vala M, et al. Genome-wide comparison of the transcriptomes of highly enriched normal and chronic myeloid leukemia stem and progenitor cell populations. Oncotarget. 2013;4(5):715-28.

20. Toofan P, Irvine D, Hopcroft L, Copland M, Wheadon H. The role of the bone morphogenetic proteins in leukaemic stem cell persistence. Biochem Soc Trans. 2014;42(4):809-15.

21. Wu X, Kabalane H, Kahli M, Petryk N, Laperrousaz B, Jaszczyszyn Y, et al. Developmental and cancer-associated plasticity of DNA replication preferentially targets GC-poor, lowly expressed and late-replicating regions. Nucleic Acids Res. 2018;46(19):10157-72.

22. Voeltzel T, Flores-Violante M, Zylbersztejn F, Lefort S, Billandon M, Jeanpierre S, et al. A new signaling cascade linking BMP4, BMPR1A, DeltaNp73 and NANOG impacts on stem-like human cell properties and patient outcome. Cell Death Dis. 2018;9(10):1011.

23. Battula VL, Le PM, Sun JC, Nguyen K, Yuan B, Zhou X, et al. AML-induced osteogenic differentiation in mesenchymal stromal cells supports leukemia growth. JCI Insight. 2017;2(13).

24. Gruber TA, Larson Gedman A, Zhang J, Koss CS, Marada S, Ta HQ, et al. An Inv(16)(p13.3q24.3)-encoded CBFA2T3-GLIS2 fusion protein defines an aggressive subtype of pediatric acute megakaryoblastic leukemia. Cancer Cell. 2012;22(5):683-97.

25. Cheng H, Ang HY, C AEF, Li P, Fang HT, Liu TM, et al. Reprogramming mouse fibroblasts into engraftable myeloerythroid and lymphoid progenitors. Nat Commun. 2016;7:13396.

26. Walkley CR, Olsen GH, Dworkin S, Fabb SA, Swann J, McArthur GA, et al. A microenvironment-induced myeloproliferative syndrome caused by retinoic acid receptor gamma deficiency. Cell. 2007;129(6):1097-110.

27. Raaijmakers MH, Mukherjee S, Guo S, Zhang S, Kobayashi T, Schoonmaker JA, et al. Bone progenitor dysfunction induces myelodysplasia and secondary leukaemia. Nature. 2010;464(7290):852-7.

28. Dong L, Yu WM, Zheng H, Loh ML, Bunting ST, Pauly M, et al. Leukaemogenic effects of Ptpn11 activating mutations in the stem cell microenvironment. Nature. 2016;539(7628):304-8.

29. Schepers K, Hsiao EC, Garg T, Scott MJ, Passegue E. Activated Gs signaling in osteoblastic cells alters the hematopoietic stem cell niche in mice. Blood. 2012;120(17):3425-35.

30. Krause DS, Lazarides K, von Andrian UH, Van Etten RA. Requirement for CD44 in homing and engraftment of BCR-ABL-expressing leukemic stem cells. Nat Med. 2006;12(10):1175-80.

31. Zhang B, Ho YW, Huang Q, Maeda T, Lin A, Lee SU, et al. Altered microenvironmental regulation of leukemic and normal stem cells in chronic myelogenous leukemia. Cancer Cell. 2012;21(4):577-92.

32. Vicente Lopez A, Vazquez Garcia MN, Melen GJ, Entrena Martinez A, Cubillo Moreno I, Garcia-Castro J, et al. Mesenchymal stromal cells derived from the bone marrow of acute lymphoblastic leukemia patients show altered BMP4 production: correlations with the course of disease. PloS one. 2014;9(1):e84496. 
33. Krause DS, Scadden DT. A hostel for the hostile: the bone marrow niche in hematologic neoplasms. Haematologica. 2015;100(11):1376-87.

34. Warfvinge R, Geironson L, Sommarin MNE, Lang S, Karlsson C, Roschupkina T, et al. Single-cell molecular analysis defines therapy response and immunophenotype of stem cell subpopulations in CML. Blood. 2017;129(17):2384-94.

35. Naka K, Hoshii T, Muraguchi T, Tadokoro Y, Ooshio T, Kondo Y, et al. TGF-betaFOXO signalling maintains leukaemia-initiating cells in chronic myeloid leukaemia. Nature. 2010;463(7281):676-80.

36. Toofan P, Busch C, Morrison H, O'Brien S, Jorgensen H, Copland M, et al. Chronic myeloid leukaemia cells require the bone morphogenic protein pathway for cell cycle progression and self-renewal. Cell Death Dis. 2018;9(9):927.

37. Grockowiak E, Laperrousaz B, Jeanpierre S, Voeltzel T, Guyot B, Gobert S, et al. Immature CML cells implement a BMP autocrine loop to escape TKI treatment. Blood. 2017;130(26):2860-71.

38. Cosset E, Hamdan G, Jeanpierre S, Voeltzel T, Sagorny K, Hayette S, et al. Deregulation of TWIST-1 in the CD34+ compartment represents a novel prognostic factor in chronic myeloid leukemia. Blood. 2011;117(5):1673-6.

39. Tipping AJ, Deininger MW, Goldman JM, Melo JV. Comparative gene expression profile of chronic myeloid leukemia cells innately resistant to imatinib mesylate. ExpHematol. 2003;31(11):1073-80.

40. Shlush LI, Mitchell A, Heisler L, Abelson S, Ng SWK, Trotman-Grant A, et al. Tracing the origins of relapse in acute myeloid leukaemia to stem cells. Nature. 2017;547(7661):104-8.

41. Raymond A, Liu B, Liang H, Wei C, Guindani M, Lu Y, et al. A role for BMP-induced homeobox gene MIXL1 in acute myelogenous leukemia and identification of type I BMP receptor as a potential target for therapy. Oncotarget. 2014;5(24):12675-93.

42. Glaser S, Metcalf D, Wu L, Hart AH, DiRago L, Mifsud S, et al. Enforced expression of the homeobox gene Mixl1 impairs hematopoietic differentiation and results in acute myeloid leukemia. Proc Natl Acad Sci U S A. 2006;103(44):16460-5.

43. Busch $\mathrm{C}$, Wheadon $\mathrm{H}$. Bone marrow niche crosses paths with BMPs: a road to protection and persistence in CML. Biochemical Society transactions. 2019;47(5):130725.

44. Bhardwaj G, Murdoch B, Wu D, Baker DP, Williams KP, Chadwick K, et al. Sonic hedgehog induces the proliferation of primitive human hematopoietic cells via BMP regulation. Nat Immunol. 2001;2(2):172-80.

45. Fuchs O, Simakova O, Klener P, Cmejlova J, Zivny J, Zavadil J, et al. Inhibition of Smad5 in human hematopoietic progenitors blocks erythroid differentiation induced by BMP4. Blood Cells Mol Dis. 2002;28(2):221-33.

46. Jeanpierre S, Arizkane K, Thongjuea S, Grockowiak E, Geistlich K, Barral L, et al. The quiescent fraction of chronic myeloid leukemic stem cells depends on BMPR1B, Stat3 and BMP4-niche signals to persist in patients in remission. Haematologica. 2020. In press.

47. Chakraborty SN, Leng X, Perazzona B, Sun X, Lin YH, Arlinghaus RB. Combination of JAK2 and HSP90 inhibitors: an effective therapeutic option in drug-resistant chronic myelogenous leukemia. Genes Cancer. 2016;7(5-6):201-8.

48. Eiring AM, Kraft IL, Page BD, O'Hare T, Gunning PT, Deininger MW. STAT3 as a mediator of BCR-ABL1-independent resistance in chronic myeloid leukemia. Leuk Suppl. 2014;3(Suppl 1):S5-6. 
49. Eiring AM, Page BDG, Kraft IL, Mason CC, Vellore NA, Resetca D, et al. Combined STAT3 and BCR-ABL1 inhibition induces synthetic lethality in therapy-resistant chronic myeloid leukemia. Leukemia. 2015;29(3):586-97.

50. Kuepper MK, Butow M, Herrmann O, Ziemons J, Chatain N, Maurer A, et al. Stem cell persistence in CML is mediated by extrinsically activated JAK1-STAT3 signaling. Leukemia. 2019;33(8):1964-77.

51. Hirsch P, Zhang Y, Tang R, Joulin V, Boutroux H, Pronier E, et al. Genetic hierarchy and temporal variegation in the clonal history of acute myeloid leukaemia. Nat Commun. 2016;7:12475.

52. Zhou HS, Carter BZ, Andreeff M. Bone marrow niche-mediated survival of leukemia stem cells in acute myeloid leukemia: Yin and Yang. Cancer Biol Med. 2016;13(2):248-59. 53. Shafat MS, Oellerich T, Mohr S, Robinson SD, Edwards DR, Marlein CR, et al. Leukemic blasts program bone marrow adipocytes to generate a protumoral microenvironment. Blood. 2017;129(10):1320-32.

54. Sharma M, Ross C, Srivastava S. Ally to adversary: mesenchymal stem cells and their transformation in leukaemia. Cancer Cell Int. 2019;19:139. 